\title{
Palatal Abscess in a Pediatric Patient: Report of a Case
}

\author{
A.Pinar Sumera \\ Peruze Celenk ${ }^{b}$
}

\section{ABSTRACT}

The palatal mass can pose a difficult diagnostic dilemma for the clinician. In differential diagnosis of the palatal mass, dental causes must be considered because they are so common. The palatal abscess typically represents the palatally directed drainage of an infection of pulpal or periodontal origin. The palatal abscess is often observed in the premolar-molar region and presents as a compressible mass or swelling usually lateral to the midline. This study reports the unusual case of a 5-year-old girl with a palatal abscess adjacent to the midline. (Eur J Dent 2008;2:291-293)

Key words: Palatal abscess; Odontogenic infections; Treatment.

\section{INTRODUCTION}

The palatal mass can pose a difficult diagnostic dilemma for the clinician. A mass or swelling of the palate can result from a periapical lesion with concomitant abscess formation, a periodontal abscess, or a neoplastic process. In differential diagnosis of the palatal mass, dental causes must

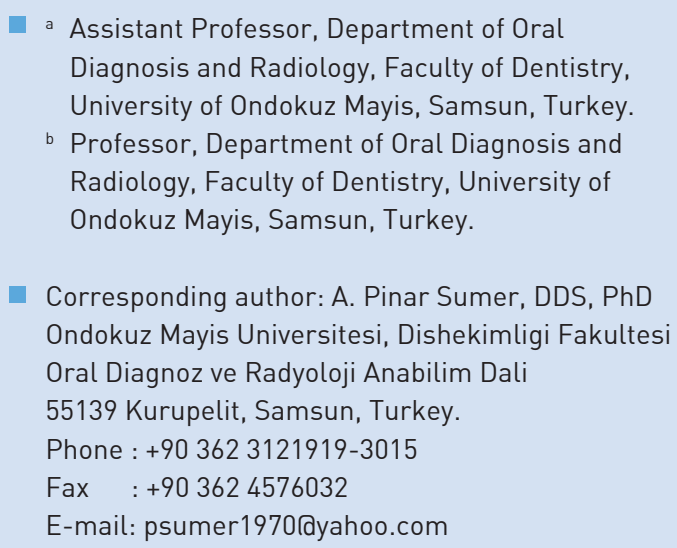

be considered because they are so common. The palate is very typical for an abscess arising from a nonvital lateral incisor or the palatal root of an upper first molar. ${ }^{2}$

An abscess is a nonspecific inflammatory response to the presence of bacteria in normally sterile tissues. The seriousness of the infection is related to the numbers and virulence of the bacteria, host resistance, and associated anatomic structures. ${ }^{3}$

This study reports an unusual case of a pediatric patient with a palatal abscess adjacent to the midline.

\section{CASE REPORT}

A 5-year-old girl presented for evaluation of painless swelling in the palate. The patient had a history of swelling in her left cheek and pain in 
the region of left posterior teeth one month ago. One week after this complaint, she had noticed a mass in her palate. She had gone a general dental practitioner. The mass was incised and antibiotics were begun, however, there was no recession in the swelling.

The patients' medical history was noncontributory. Extraoral examination revealed a mild swelling in the left cheek. Palpable left submandibular lymph nodes were present. On intraoral examination, dental caries in posterior teeth and a $2 \times 3 \mathrm{~cm}$, regular, rounded swelling in the palate adjacent to the midline was observed (Figure 1). The swelling did not show signs of fluctuation and overlying mucosa is normal and there is no ulceration. Clinically, a sinus in the buccal mucosa of the upper left second primary molar was observed.

Both periapical and panoramic radiographs showed caries in the upper left second primary molar without periapical radiolucency (Figure 2). The patients enrolled for the extraction of her upper left second primary molar tooth. The patient recovered completely after the extraction (Figure 3).

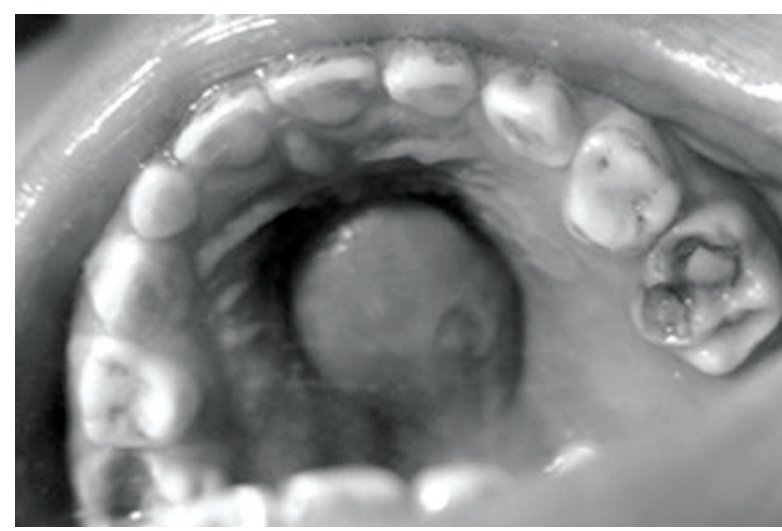

Figure 1. Clinical view of the palatal abscess adjacent to the midline.

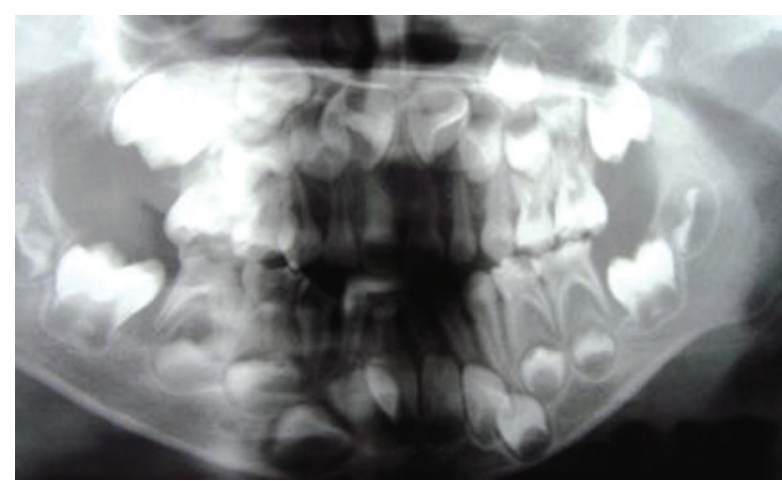

Figure 2. Panoramic radiography shows caries in the upper left second primary molar without periapical radiolucency.

\section{DISCUSSION}

Most infections of the oral cavity are primary odontogenic infections originate within the dental pulp (most commonly), periodontal tissue or pericoronal tissue. ${ }^{4}$ Odontogenic infections may spread from the affected tooth along the path of least resistance into anatomic spaces far removed from the site of initial infection. ${ }^{5,6}$ The palatal abscess typically represents the palatally directed drainage of an infection of pulpal or periodontal origin. The most common source is from the involvement of a palatal root of a maxillary molar tooth. ${ }^{1}$

The palatal abscess is often observed in the premolar-molar region and presents as a compressible mass or swelling usually lateral to the midline. ${ }^{1}$ This palatal abscess is an unusual one that localized adjacent to the midline of the palate.

Definitive treatment for odontogenic abscesses begins with either extraction or a pulpectomy of the offending tooth and antibiotic therapy is important. The principle of treatment of the palatal abscess is the same as for any abscess: drainage must be established after the identification of the source of the infection. ${ }^{1}$ Carious left upper primary second molar was extracted in the present case.

The palatal abscess is commonly observed lateral to the midline and it is easy to diagnose it. However, in rare cases palatal abscess can be localized adjacent to the midline and can pose a difficult diagnostic dilemma for the clinician. The taking of the history and intraoral examination are valuable diagnostic tools in conjunction with radiographic examination for the evaluation of palatal abscess.

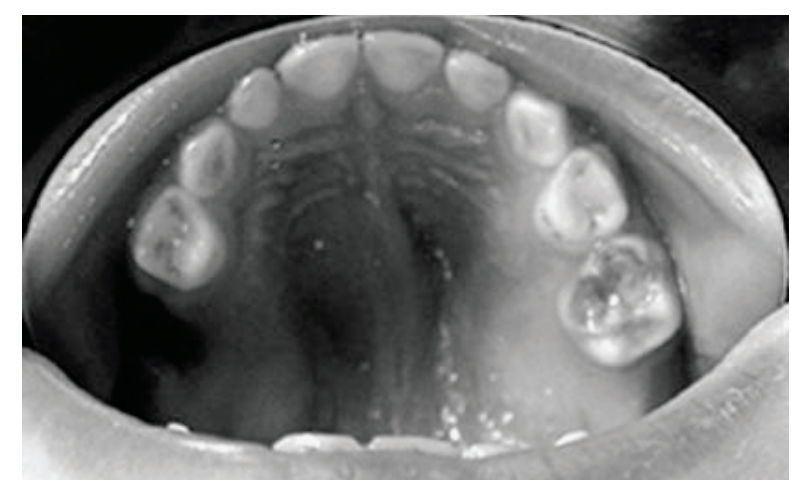

Figure 3. Clinical view of the palate after extraction. 


\section{REFERENCES}

1. Houston GD, Brown FH. Differential diagnosis of the palatal mass. Compendium 1993;14:1222-1224.

2. Odell EW. Clinical Problem solving in dentistry. 2nd ed. Elsevier Science, 2004:223-226.

3. Hargreaves KM, Goodis HE. Seltzer and Bender's dental pulp. $3^{\text {rd }}$ ed. Quintessence Publishing, 1984.

4. Maestre Vera JR. Treatment options in odontogenic infection. Med Oral Patol Oral Cir Bucal 2004;9:19-31.

5. Mitchell CS, Nelson Jr MD. Orofacial abscess of odontogenic origin in the pediatric patient: Report of two cases. Pediatr Radiol 1993;23:432-434.

6. Jimenez Y, Bagan JV, Murillo J, Poveda R. Odontogenic infections. Complications. Systemic manifestations. Med Oral Patol Oral Cir Bucal 2004;9:139-147. 